\title{
LACUNARITY ANALYSES OF MULTIFRACTAL AND NATURAL GRAYSCALE PATTERNS
}

\author{
ANKUR ROY* and EDMUND PERFECT ${ }^{*, \dagger, \ddagger}$ \\ *Department of Energy Resources Engineering \\ Stanford University, Stanford, CA 94305-2220, USA \\ ${ }^{\dagger}$ Department of Earth and Planetary Sciences \\ University of Tennessee, Knoxville, TN 37996-1410, USA \\ ‡eperfect@utk.edu
}

Received January 24, 2014

Accepted May 1, 2014

Published September 5, 2014

\begin{abstract}
Lacunarity $(L)$ is a scale $(r)$-dependent parameter that was developed for quantifying clustering in fractals and has subsequently been employed to characterize various natural patterns. For multifractals it can be shown analytically that $L$ is related to the correlation dimension, $D_{2}$, by: $d \log (L) / d \log (r)=D_{2}-2$. We empirically tested this equation using two-dimensional multifractal grayscale patterns with known correlation dimensions. These patterns were analyzed for their lacunarity using the gliding-box algorithm. $D_{2}$ values computed from the $d \log (L) / d \log (r)$ analysis gave a $\sim 1: 1$ relationship with the known $D_{2}$ values. Lacunarity analysis was also employed in discriminating between multifractal grayscale patterns with the same $D_{2}$ values, but different degrees of scale-dependent clustering. For this purpose, a new lacunarity parameter, $\langle L\rangle$, was formulated based on the weighted mean of the log-transformed lacunarity values at different scales. This approach was further used to evaluate scale-dependent clustering in soil thin section grayscale images that had previously been classified as multifractals based on standard method of moments box-counting. Our results indicate that lacunarity analysis may be a more sensitive indicator of multifractal behavior in natural grayscale patterns than the standard approach. Thus, multifractal behavior can be checked without having to compute the whole spectrum of non-integer dimensions, $D_{q}(-\infty<q<+\infty)$ that typically characterize a multifractal. The new $\langle L\rangle$ parameter should be useful to researchers who want to explore
\end{abstract}

${ }_{\ddagger}^{\ddagger}$ Corresponding author. 
the correlative influence of clustering on flow and transport in grayscale representations of soil aggregates and heterogeneous aquifers.

Keywords: Lacunarity; Clustering; Correlation Dimension; Multifractals; Gliding-Box Algorithm; Soil Structure.

\section{NOMENCLATURE}

$D_{2}$ : correlation dimension

$E$ : Euclidean dimension

$b, p$ : scale-factor and probability of retaining a cell in a generator - terms related to generating multifractal patterns

$L$ : lacunarity

$\langle L\rangle$ : weighted mean of log-transformed lacunarity

$r, r_{t}$ : length scale at which lacunarity is determined and maximum value of $r$ i.e. total length of a pattern under investigation

$s(r)$ : mass of pixels within a box of length, $r$

$\overline{s(r)}$ : mean of distribution of masses recorded with box-length, $r$

$s_{s}^{2}(r)$ : variance of distribution of masses recorded with box-length, $r$

\section{INTRODUCTION}

Lacunarity is a parameter that characterizes the distribution of spaces or gaps in a pattern as a function of scale. Originally proposed for distinguishing between monofractal patterns having the same fractal dimension but different degrees of clustering, ${ }^{1}$ lacunarity has since been used for analyzing scaledependent clustering in natural binary datasets, both fractal and non-fractal. Relevant examples include rock fracture networks, ${ }^{2}$ soil pore space geometry, ${ }^{3,4}$ thresholded medical images ${ }^{5,6}$ and various ecological patterns. ${ }^{7}$ Lacunarity can also be used to quantify clustering in multifractal and natural non-binary (grayscale) patterns. ${ }^{8-11}$ Lacunarity analyses of grayscale fields are now common in the medical imaging literature [e.g. Refs. 12-14], but this approach does not seem to have been applied previously to geometrical multifractals or soil thin section images.

The gliding-box algorithm can be used to quantify the lacunarity, $L$, at a given scale (or box-size, $r)$. Since values vary with box-size, the results are generally reported in terms of the function: $L(r)$. It has been theoretically established that when logtransformed values of $L$ and $r$ are plotted, both fractals and multifractals result in straight lines such that the slope plus the known embedding dimension equals the box or correlation dimension, respectively. ${ }^{15}$

Although there have been a few lacunarity studies of both synthetic and natural multifractal patterns, ${ }^{7,16}$ there is not enough evidence to unequivocally establish that lacunarity analysis can delineate multifractal behavior of non-binary data, and that the true correlation dimension, $D_{2}$, of a multifractal can be found by employing this technique. In the present paper, we test Allain and Cloitre's theory ${ }^{15}$ using multifractal grayscale patterns with known correlation dimensions. We also propose a novel technique for expressing lacunarity as a single number that takes into account the clustering of elements in a pattern at different scales thus differentiating it from other patterns having the same correlation dimension but different textures. Since lacunarity is a general technique applicable to all grayscale and binary patterns, this number may also be used for calculating a distance between any two patterns, fractal, multifractal or Euclidean. It can thus serve as a quantitative measure for finding the difference between patterns in general. In a final application, we focus on natural grayscale images (in the form of soil thin sections) that have been previously analyzed as multifractals using the more standard method of moments approach based on box counting (BC) ${ }^{17}$ and show that lacunarity analysis may be a more reliable technique for establishing multifractal behavior.

\section{LACUNARITY AS AN INDICATOR OF MULTIFRACTAL BEHAVIOR}

\subsection{Multifractal Grayscale Patterns}

A set of two-dimensional multifractal grayscale patterns were constructed following the steps outlined in Ref. 18, and subsequently employed by other researchers. ${ }^{19,20}$ The process is akin to generating a Sierpinski carpet, only the zeros and ones in the pattern are replaced with fractional values. The 


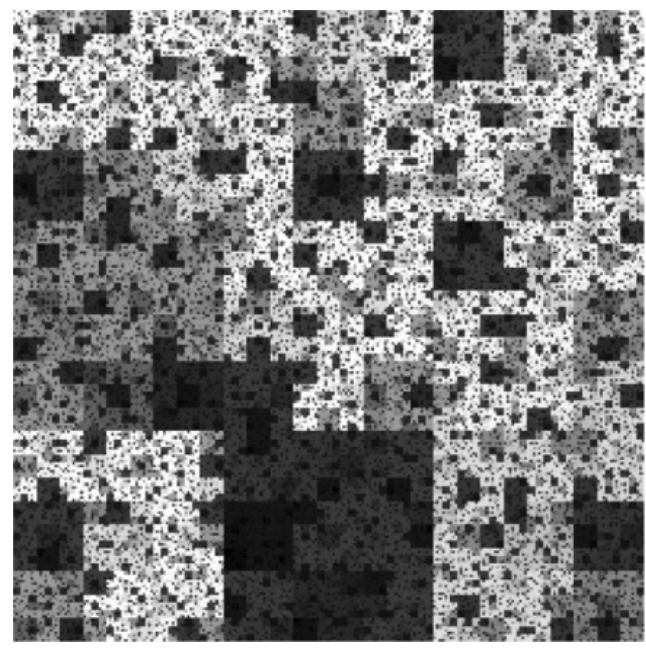

Fig. 1 A grayscale random multifractal pattern: $p 8 r 3$. Lighter phases have higher mass fractions.

algorithm involves normalizing mass-fractions calculated from the truncated binomial distribution for an average probability, $p$, of retaining a cell in the generator with a scale factor, $b$, in successive iterations. As described in Ref. 19 the locations of the normalized mass fractions can be spatially randomized so as to create a random geometrical multifractal grayscale pattern (Fig. 1). Eight different patterns were created from a generator with a scale factor of $b=3$ and probability values corresponding to $p=1 / 9,2 / 9,3 / 9,4 / 9,5 / 9,6 / 9,7 / 9$ and $8 / 9$ by iterating the system 5 times to produce grayscale fields of size $3^{5} \times 3^{5}$ pixels. For each of the 8 models (as seen in Fig. 1 in Ref. 19), 3 random realizations were constructed amounting to a total of 24 random multifractal grayscale patterns. These were named using the following scheme: $p \# r$ \#\# where \# corresponds to the numerator of the $p$-value (1-8) and \#\# to the realization $(r)$ number $(1-3)$. For example, a pattern from the model $p=3 / 9$ and random realization 2 will be named $p 3 r 2$. Figure 1 corresponds to the pattern $p 8 r 3$.

\subsection{Lacunarity of Grayscale Patterns}

Lacunarity is a scale-dependent measure of textural heterogeneity that can be quantified by employing the gliding-box algorithm. ${ }^{15,21}$ Essentially, this method involves sliding a window or an interrogator box of a given length, $r$, translated in increments of a chosen unit length (usually that of a pixel) across the whole pattern such that the total number of steps is given by $\left(r_{t}-r+1\right)^{2}, r_{t}$ being the length of the entire pattern. The details of this technique employed in analyzing binary patterns can be found in Ref. 2. In the case of grayscale patterns, the mass of all pixels, $s(r)$, contained within the interrogator box at each step is calculated and a distribution of this mass at the scale $r$ is obtained by gliding the box through all the steps. Finally, the mean, $s(r)$ and variance, $s_{s}^{2}(r)$ of this distribution are used in calculating the lacunarity, $L(r)$, at the scale $r$ as:

$$
L(r)=s_{s}^{2}(r) /[\overline{s(r)}]^{2}+1 .
$$

Typically, $L(r)$ is calculated for a range of boxsizes, and for any given pattern, this curve will have upper and lower bounding values. It can be easily perceived that at $r=1$, the interrogator box contains only 1 pixel and it moves $r t^{2}$ steps equal to the total number of pixels (each associated with a mass number) in the pattern. It then follows that $L(1)$ is the same for patterns with the same set of mass numbers but distributed differently in space. In other words, all random realizations of a model corresponding to a specific $p$-value will have the same maximum lacunarity, $L(1)$. At $r=r_{t}$, there is only one box that covers the entire pattern and hence the distribution of masses, $s\left(r_{t}\right)$ consists of just one value. This implies that the variance, $s_{s}^{2}\left(r_{t}\right)=0$ such that the lacunarity is $L\left(r_{t}\right)=1$. It may be noted that uniform patterns have no variance and hence they furnish, $L(r)=1$ for all $r$ values.

\subsection{Lacunarity and the Correlation Dimension}

It has been theoretically demonstrated in Ref. 15 that in the case of multifractals, lacunarity, $L(r)$ is related to the size of the interrogator box, $r$ by a power-law such that the power-term equals $D_{2}-E$, where $D_{2}$ is the correlation dimension and $E$ the Euclidean embedding dimension. Based on this result, it is expected that the log-transformed lacunarity functions for the two-dimensional multifractal patterns described in Sec. 2.1 can be fitted with a straight line of the form, $y=m x+c$, such that the slope is:

$$
m=D_{2}-2 .
$$

For each of the 24 multifractal patterns, the $\log L(r)$ versus $\log r$ values were calculated and a subset of the array of points thus obtained was fitted with a straight line. Figure 2 a shows one such plot for the pattern $p 8 r 3$. Figure $2 \mathrm{~b}$ shows the local slope of the $\log L(r)$ versus $\log r$ curve calculated at each $r$ with the dashed line describing the slope 


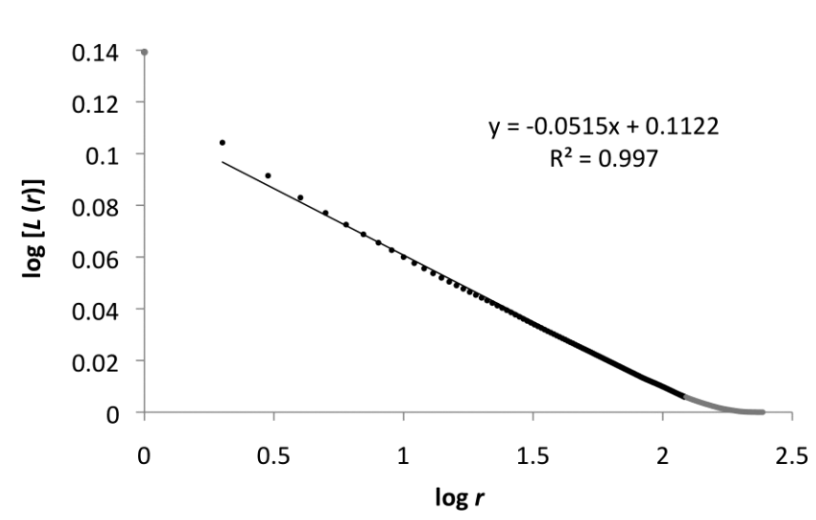

(a)

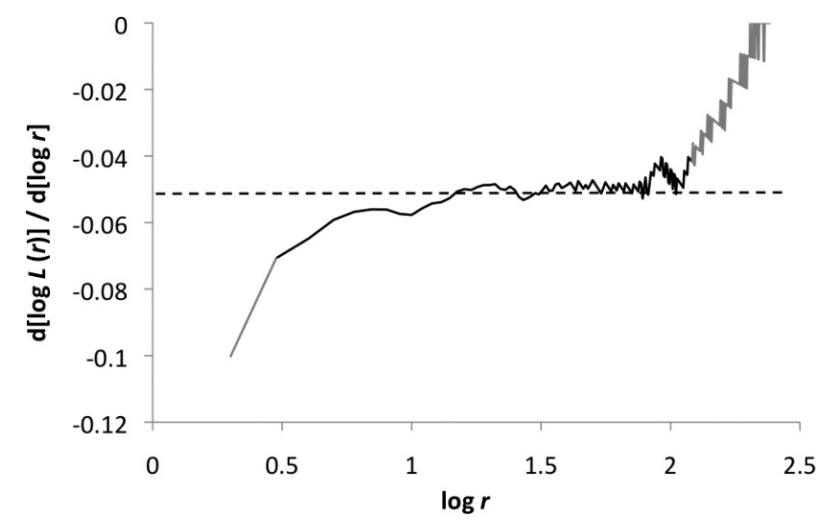

(b)

Fig. 2 (a) $\log L(r)$ versus $\log r$ plot for pattern $p 8 r 3$ : points not included in the linear fit are shown in gray. (b) Local slope of (a) plotted as $d[\log L(r)] / d[\log r]$ against $\log r$ : dashed line shows slope of linear fit from (a), the "flat segment" used for fitting the linear model is shown in black.

of the fitted line from Fig. 2a. In order to meet Allain and Cloitre's condition of multifractality, ${ }^{15}$ and to estimate the proper correlation dimension for the pattern, only the relatively "flat" segment of the curve in Fig. 2b was considered for fitting a linear model to the points in Fig. 2a. Also, it is important to realize here that patterns that do not furnish such "flat" segments are thus not multifractals. We will come back to a detailed discussion on this in a later section. For large $r$ values it is seen from Fig. $2 b$ that the local slope starts to fluctuate and increases abruptly. Therefore, the points corresponding to box-sizes $r>r_{t} / 2$ were not included in the fitting. For smaller $r$ values, the coefficient of determination, $R^{2}$, of the fit improved when points were sequentially excluded from the left in Fig. 2a. However, it is the first point at $r=1$ that mostly influences the change in slope such that there was a difference of $\sim 4 \%$ in the estimated slope if this point was excluded from the fit. If two or more additional points were excluded from the fitting there was no more than $\sim 1 \%$ difference. This is mainly because the first few points on the left hand side of the $\log L(r)$ versus $\log r$ plot were always sparsely distributed. Therefore from the lower end, only the first point in the plot was excluded from the fitting. The points thus excluded from both ends are shown in gray in Fig. 2.

Correlation dimensions for all 24 patterns were calculated from their $\log L(r)$ versus $\log r$ plots by employing the above protocol. An average estimated $D_{2}$ value from all three realizations of each model corresponding to a particular $p$-value was computed and compared to the theoretical $D_{2}$

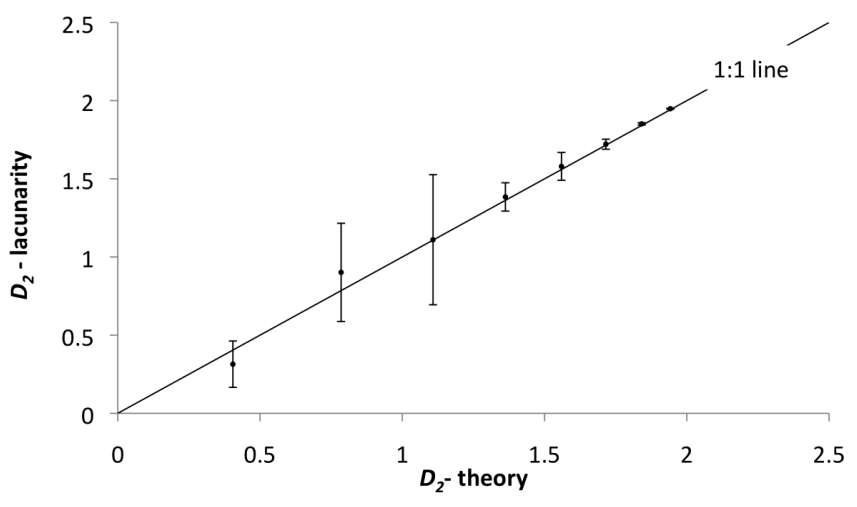

Fig. 3 Correlation dimension, $D_{2}$, estimated from lacunarity analysis compared to theoretical $D_{2}$ values for random multifractal fields with scaling factor $b=2$, and probability of occupied cells, $p=1 / 9$ to $8 / 9$ (three realizations for each of the eight fields); average values of 3 realizations with bars corresponding to the $95 \%$ confidence intervals.

value. ${ }^{18}$ The results are graphed in Fig. 3. The $95 \%$ confidence intervals in the computed $D_{2}$ values arising from the 3 random realizations for each model are shown as vertical bars. As can be seen from the figure, the computed $D_{2}$ values overlap the 1:1 line $\left(45^{\circ}\right.$ slope) and are thus are statistically equal to their theoretical counterparts. This analysis demonstrates empirically that an almost exact value of the correlation dimension for multifractals can be found from lacunarity analysis. It may therefore be concluded that lacunarity analysis can detect if a grayscale pattern exhibits multifractal behavior (on the basis of log-log linearity) and, if it does, provide an accurate estimate its correlation dimension (from the slope of this relation). 


\section{LACUNARITY AS A MEASURE OF CLUSTERING IN MULTIFRACTALS}

Lacunarity analysis has been used as a measure of clustering in monofractals and other binary patterns. Examples include those of simulated landscape maps $^{21}$ and fracture networks. ${ }^{2}$ Lacunarity has also been used for textural analysis of grayscale patterns. ${ }^{8-14}$ This section extends the previous research by implementing lacunarity as a measure of clustering in geometrical multifractal grayscale patterns.

A set of three deterministic (Figs. 4a-4c) and one random (Fig. 4d) multifractal grayscale patterns of size $343 \times 343$ pixels were constructed using the following parameters: $b=7, p=40 / 49$ and 3 iterations. These patterns will henceforth be referred to as models A, B, C and D, respectively. The random multifractal grayscale pattern D was generated using exactly the same approach as described in Sec. 2.1. The deterministic patterns $(\mathrm{A}-\mathrm{C})$ were generated by placing nine of the largest mass-fractions obtained from the truncated binomial distribution ${ }^{18}$ in different predetermined locations within the generator and the rest of the 40 mass-fraction numbers in random locations around them, and then iterating the system 3 times. Of these four patterns, $\mathrm{A}$ and $\mathrm{C}$ are the least and most clustered, respectively, and may be thought of as multifractal counterparts of Figs. 3b-7b and 3a7a, respectively in Ref. 22. Patterns B and D are intermediately clustered. A fifth model, designated E (Fig. 4e), was constructed by completely randomizing the spatial locations of the mass fractions produced by $b=7, p=40 / 49$ and 3 iterations multifractal model; it is thus a true random grayscale pattern. As such, it should have minimal clustering, and negligible lacunarity.

Models A through E were analyzed for their lacunarity using the gliding box method and the resulting $\log L(r)$ versus $\log r$ values plotted as before (Fig. 5). All of the multifractal models produced a straight line trend that was very different from that of model E, which is not a multifractal. Being a truly random pattern, the lacunarity values for model $\mathrm{E}$ follow a nonlinear curve and quickly converge to a value of zero which shows that at larger scales $(r>30)$ it behaves like a uniform pattern

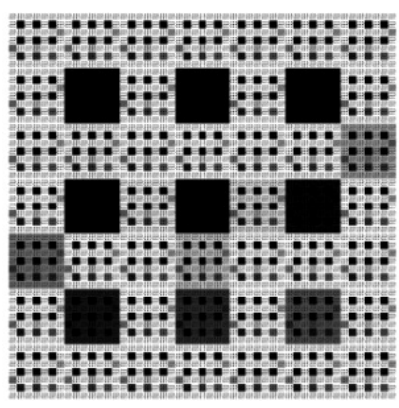

(a)

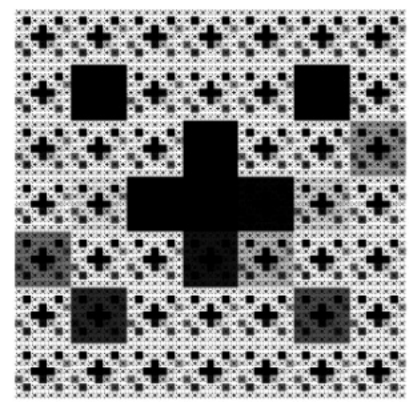

(b)

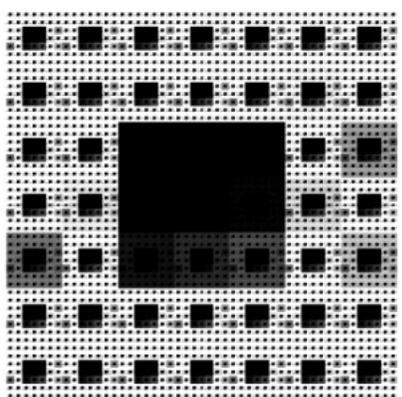

(c)

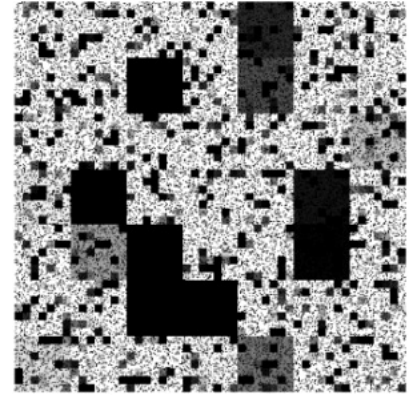

(d)

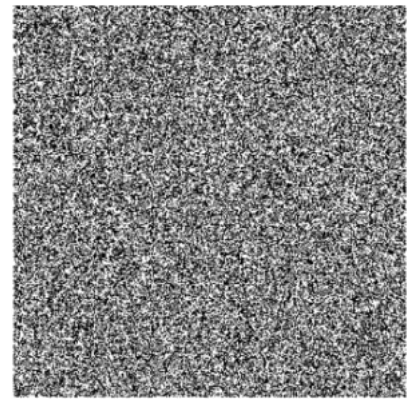

(e)

Fig. 4 Grayscale multifractal models with $b=7$ and $p=40 / 49$, and 3 iterations; models (a), (b), (c): deterministic multifractals showing low, medium and high clustering, respectively; model (d): random multifractal; model (e): non-multifractal random grayscale pattern with same mass fractions as the others models. 


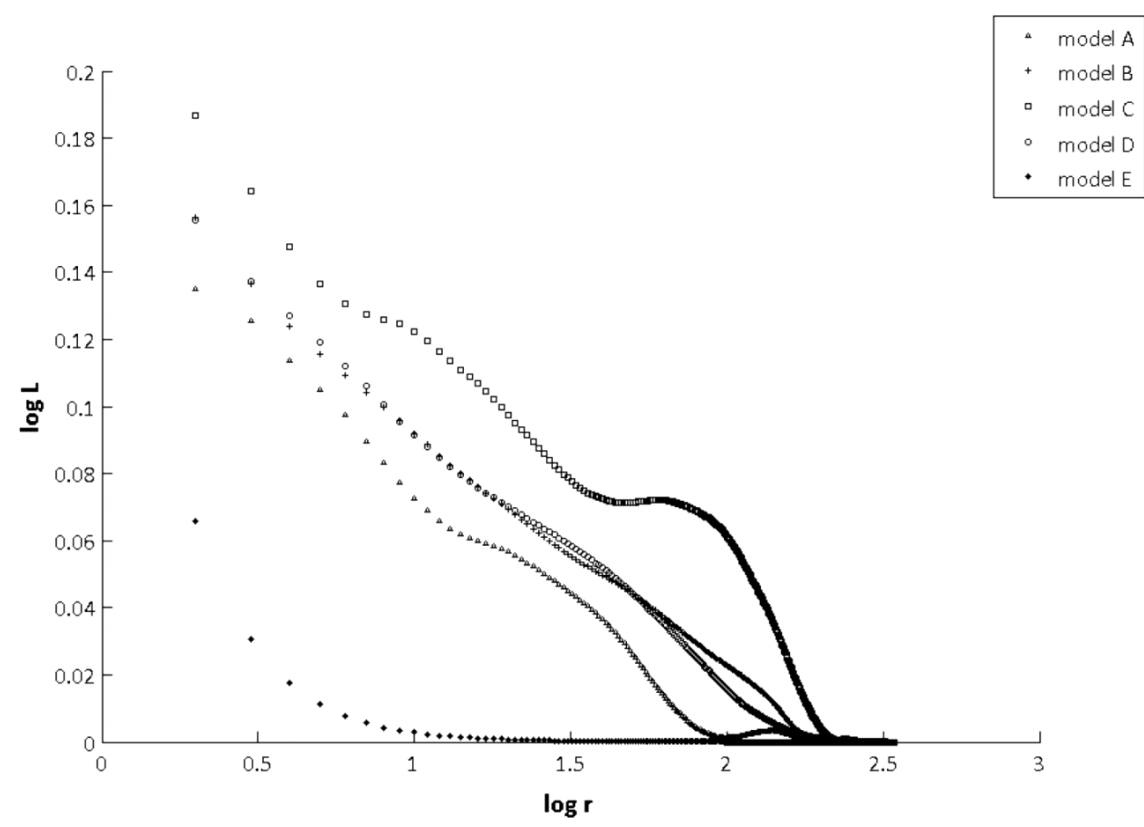

Fig. $5 \log L(r)$ versus $\log r$ plots for models A-E. Note curvilinear behavior of model E (random), as compared to the other (multifractal) models.

with $L(r)=1($ since $\log L(r)=0)$ at all $r$ values. Despite some kinks in the linearity of the $\log -\log$ plots for the three deterministic multifractal grayscale patterns, created by the non-random geometric arrangement of the generator mass fractions, regression analysis still yielded estimates of $D_{2}$ (Table 1) that were within $1 \%$ of the theoretical value of 1.914. As expected, the random multifractal grayscale pattern D produced the most linear relationship between $\log L(r)$ and $\log r$ resulting in the best estimate of $D_{2}$ (within $0.5 \%$ of theoretical value).

Amongst the multifractals, model A always had the lowest lacunarity values while model $\mathrm{C}$ had the highest (Fig. 5). Models B and D had intermediate lacunarity values at all scales. These results are

Table 1 Correlation Dimensions $\left(D_{2}\right)$, Associated Coefficients of Determination $\left(R^{2}\right)$ and Weighted Mean Log-Transformed Lacunarities, $\langle L\rangle$, for the Five Synthetic Grayscale Fields Illustrated in Fig. 4.

\begin{tabular}{cccr}
\hline Model & $\boldsymbol{D}_{\mathbf{2}}$ & $\boldsymbol{R}^{\mathbf{2}}$ & $\langle\boldsymbol{L}\rangle^{\mathbf{a}}$ \\
\hline A & 1.932 & 0.947 & 6.1 \\
B & 1.928 & 0.996 & 12.6 \\
C & 1.928 & 0.915 & 25.7 \\
D & 1.922 & 0.995 & 11.1 \\
E & n.a. & n.a. & 0.1 \\
\hline
\end{tabular}

${ }^{\mathrm{a}}\langle L\rangle$ values reported as $\times 10^{3}$

n.a. $=$ not applicable $(\log L(r)$ versus $\log r$ plot nonlinear $)$ consistent with the different degrees of clustering imposed on the patterns by the choice of the locations of mass fractions in the different generators (Fig. 4). For purposes of comparison between patterns with different clustering, it can be advantageous to report lacunarity as a single number rather than a $\log L(r)$ versus $\log r$ plot. In such plots, $L(r)$ is high at small values of $r$, while for large values of $r, L(r)$ is low and, as seen in Fig. 5, the differences in lacunarity between variously clustered grayscale multifractals are most pronounced in the middle order $r$ values. A log-weighted mean of the log-transformed values of lacunarity, $\langle L\rangle$, will best describe these differences while taking into account the variability in $L(r)$ with $r$. This weighted mean can be calculated with the following equation:

$$
\langle L\rangle=\frac{\sum[\log L(r)][\log r]}{\sum \log r} .
$$

The values of $\langle L\rangle$ for models $\mathrm{A}$ through $\mathrm{E}$ are given in Table 1. It may be noted that the different patterns rank as $\mathrm{E} \ll \mathrm{A}<\mathrm{D} \sim \mathrm{B}<\mathrm{C}$ in terms of $\langle L\rangle$ as expected from visual inspection of the different degrees of clustering in Fig. 4.

\section{LACUNARITY ANALYSIS OF NATURAL GRAYSCALE IMAGES}

In this final section, we apply the lacunarity technique developed above to a set of natural grayscale 


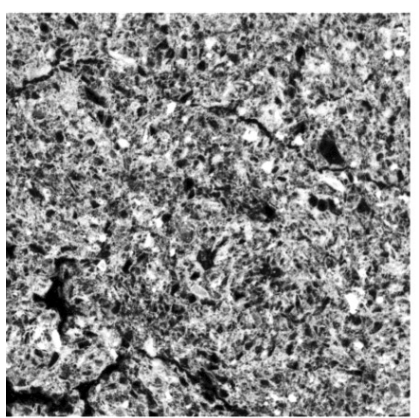

Soil 1

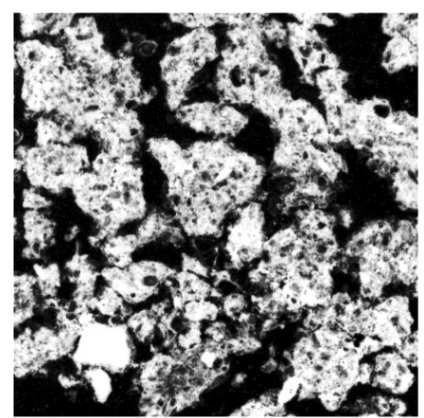

Soil 2

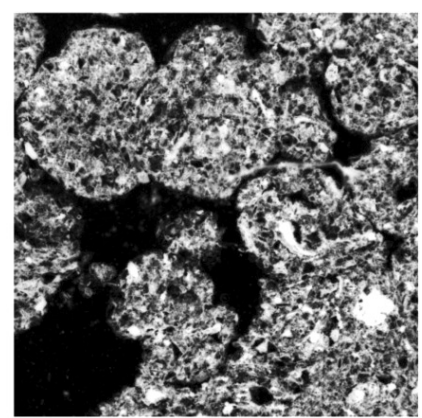

Soil 3

Fig. 6 Natural grayscale images of soil thin sections from Zhou et al. (2011).

Note: Soil 1 is comparable to model E in Fig. 4, while soil 3 is comparable to model D.

patterns that have been previously characterized as multifractals based on the method of moments BC approach. ${ }^{17}$ These patterns comprise a set of three soil thin sections photographed in plane polarized light with a Nikon DS-Fi1 digital camera at an 8-bit depth resolution (Fig. 6). Each image is $1024 \times 1024$ pixels, the length of a pixel being $1.9 \mu \mathrm{m}$. As described in Ref. 17, Soil 1 (Fig. 6a) has a relatively homogenous structure, soil 2 (Fig. 6b) has medium-sized aggregates with evenly distributed pores, while soil 3 (Fig. 6c) has well-developed aggregates with large pores. Visual inspection of these patterns suggests that soil 1 is comparable to model $\mathrm{E}$ from the previous section, while soil 3 compares to most closely to model D.
The $\log L(r)$ versus $\log r$ plots for these soils are shown in Fig. 7a. Figure 7b shows the local slopes of the $\log L(r)$ versus $\log r$ curves calculated at each $r$ and denoted by $d(\log L) / d(\log r)$. It can be seen from this figure that only soil 3 behaves like the multifractal pattern $p 8 r 3$ in Fig. $2 \mathrm{~b}$ and displays somewhat of a "flat segment" in the $d(\log L) / d(\log r)$ versus $\log r$ plot. It therefore meets the condition of multifractality as stated in Ref. 15 over the corresponding range of $r$ values and can thus be classified as a true multifractal pattern. Soil 2 clearly does not have any "flat segments" and is therefore not a multifractal. Soil 1 reaches a constant $L(r)$ of one (i.e. $\log L(r)=0)$ around $r=100$. Therefore at larger scales, soil 1 behaves like the pure random pattern,

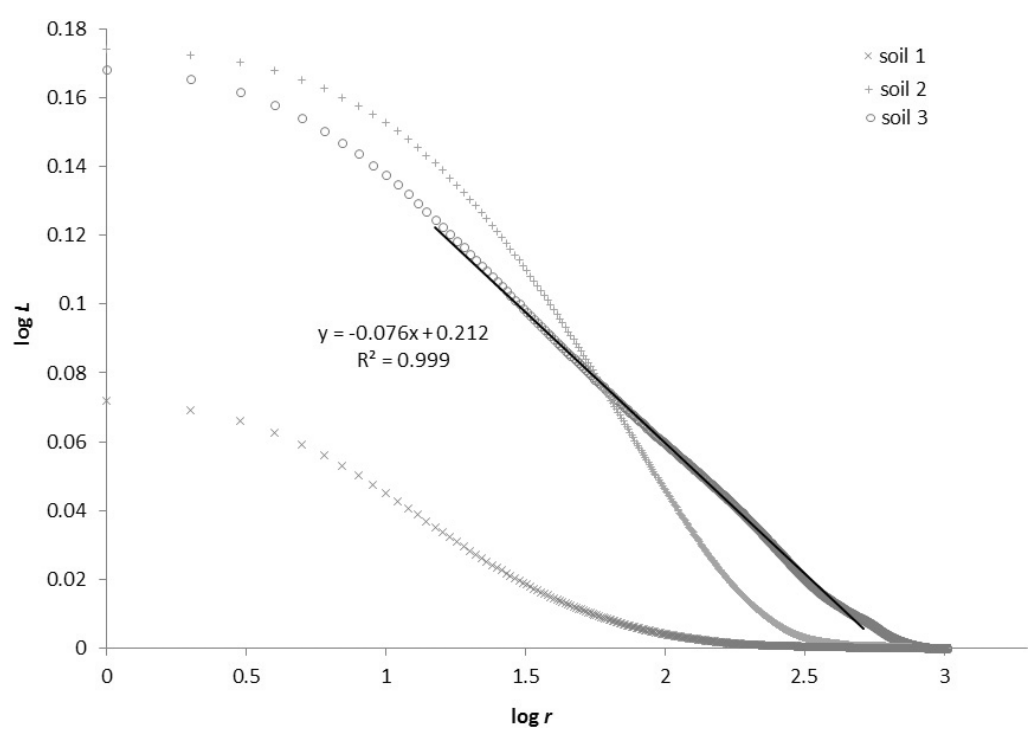

(a)

Fig. 7 (a) $\log L(r)$ versus $\log r$ plots for natural grayscale images. The data for soil 3 can be fitted by a straight line between scales, $r=15$ and $r=512$ exhibiting multifractal behavior over that range; soil 1 is comparable to the $L(r)$ versus log $r$ plot for model E (random) in Fig. 5; soil 2 is intermediate. (b) Local slopes of soils from (a) plotted as $d[\log L(r)] / d[\log r]$ against $\log r$ : dotted line shows slope of linear fit of soil 3 from (a). 


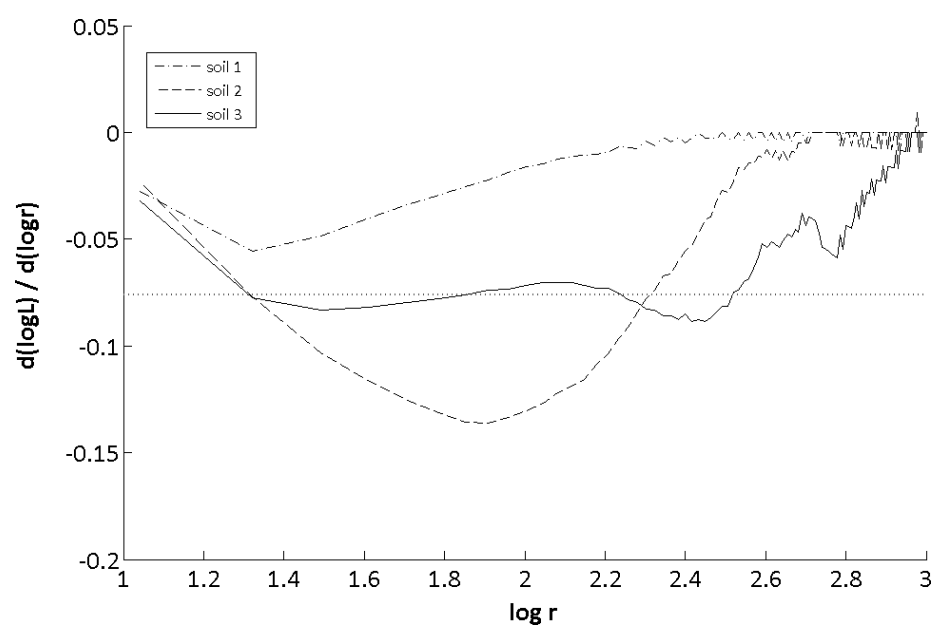

(b)

Fig. 7 (Continued)

model E, which is expected due to its "relatively homogenous structure".

Based on standard BC analyses, it was concluded that all three soil thin section images were multifractal. ${ }^{9}$ It is evident from the above results, however, that only soil 3 can be considered truly multifractal in nature, with a linear $L(r)$ versus $\log r$ plot covering $\sim 2$ orders of magnitude. Thus, compared to the BC method, lacunarity analysis appears to be more reliable when it comes to establishing multifractal behavior of natural grayscale patterns. A similar conclusion was arrived from Ref. 23 when comparing the $\mathrm{BC}$ and gliding box approaches for conducting full multifractal analyses. The gliding box algorithm, which forms the basis of lacunarity analysis, produces more continuous coverage with a large number of $r$ values as compared to $\mathrm{BC}$, which relies on fewer discrete step increments.

The weighted mean log-transformed lacunarities, $\langle L\rangle$, of the three soils, along with the correlation

Table 2 Correlation Dimensions $\left(D_{2}\right)$, Associated Coefficients of Determination $\left(R^{2}\right)$ and Weighted Mean Log-Transformed Lacunarities, $\langle L\rangle$, for the Three Natural Grayscale Fields Illustrated in Fig. 6.

\begin{tabular}{cccr}
\hline Soil & $\boldsymbol{D}_{\mathbf{2}}$ & $\boldsymbol{R}^{\mathbf{2}}$ & $\langle\boldsymbol{L}\rangle^{\mathbf{a}}$ \\
\hline 1 & n.a. & n.a. & 1.2 \\
2 & n.a. & n.a. & 8.4 \\
3 & 1.924 & 0.999 & 15.5 \\
\hline
\end{tabular}

${ }^{\mathrm{a}}\langle L\rangle$ values reported as $\times 10^{3}$

n.a. $=$ not applicable $(\log L(r)$ versus $\log r$ plot nonlinear $)$ dimension, $D_{2}$, for soil 3 as computed from the $\log L(r)$ versus $\log r$ plot, are reported in Table 2 . The $D_{2}$ value is within $\sim 2 \%$ of the value for soil 3 based on $\mathrm{BC}^{9}{ }^{9}$ The $\langle L\rangle$ values show that soil 3 is the most clustered amongst the three thin sections and this is no surprise since it has was previously reported as having "relatively well-developed aggregates with large pores". Also, the $\langle L\rangle$ value for soil 3 compares favorably with that of model D in Table 1.

\section{CONCLUSIONS}

The results from our research on lacunarity analysis of natural and multifractal grayscale patterns bring forth three main points. First, they empirically test Allain and Cloitre's equation ${ }^{15}$ that relates lacunarity to the correlation dimension in grayscale patterns. It is demonstrated that by running a lacunarity analysis on a grayscale pattern one can check for possible multifractal behavior and hence find the correlation dimension. Second, they show that lacunarity analysis can delineate clustering in synthetic and natural grayscale images regardless of their multifractal nature. Third, and most importantly, this work introduces the novel concept of expressing lacunarity as a single number that takes into account the clustering in a pattern at different scales. This new lacunarity parameter is particularly helpful for comparing different grayscale patterns or images in terms of their clustering attributes. It will likely prove useful in future research exploring the influence of texture on flow and transport processes within grayscale 
representations of soil aggregates and heterogeneous aquifers.

\section{REFERENCES}

1. B. B. Mandelbrot, The Fractal Geometry of Nature, 1st edn. (Freeman, New York, NY, 1983), p. 468.

2. A. Roy, E. Perfect, W. M. Dunne, N. E. Odling and J. W. Kim, Lacunarity analysis of fracture networks: Evidence for scale-dependent clustering, J. Struct. Geology 32 (2010) 1444-1449.

3. L. Luo and H. Lin, Lacunarity and fractal analyses of soil macropores and preferential transport using micro-x-ray computed tomography, Soil Sci. Soc. Am. J. 8 (2008) 233-241.

4. H. C. Chun, D. Giménez and S. W. Yoon, Morphology, lacunarity and entropy of intra-aggregate pores: Aggregate size and soil management effects, Geoderma 146(1-2), (2008) 83-93.

5. G. Dougherty and G. M. Henebry, Lacunarity analysis of spatial pattern in CT images of vertebral trabecular bone for assessing osteoporosis, Med. Eng. Phys. 24 (2002) 129-138.

6. F. Yasar and F. Akgunlu, Fractal dimension and lacunarity analysis of dental radiographs, Dentomaxillofac. Radiol. 34(5) (2005) 261-267.

7. R. E. Plotnick, R. H. Gardner, W. W. Hargrove, K. Prestegaard and M. Perlmutter, Lacunarity analysis: A general technique for the analysis of spatial patterns, Phys. Rev. E. 53(5) (1996) 5461-5468.

8. S. W. Myint and N. Lam, A study of lacunarity based texture analysis approaches to improve urban image classification, Comput. Environ. Urban Syst. 29 (2005) 501-523.

9. G. Du and T. S. Yeo, A novel lacunarity estimation method applied to SAR image segmentation, IEEE Trans. Geoscience Remote Sens. 40(12) (2009) 2687-2691.

10. A. R. Backes, A new approach to estimate lacunarity of texture images, Pattern Recognit. Lett. 34(13) (2013) 1455-1461.

11. N. A. Valous, D.-W. Sun, P. Allen and F. Mendoza, The use of lacunarity for visual texture characterization of pre-sliced cooked pork ham surface intensities, Food Res. Int. 43 (2010) 387-395.
12. D. C. Manikka-Baudge and G. Dougherty, Texture analysis using lacunarity and average local variance, Proc. SPIE 7259 (2009) 725953, 1-12.

13. I. Pantic, L. Harhaji-Trajkovic, A. Pantovic, N. T. Milosevic and V. Trajkovic, Changes in fractal dimension and lacunarity as early markers of UVinduced apoptosis, J. Theor. Biol. 303 (2012) 87-92.

14. F. Soares, F. Janela, M. Pereira, J. Seabra and M. M. Freire, 3D Lacunarity in multifractal analysis of breast tumor lesions in dynamic contrastenhanced magnetic resonance imaging, IEEE Trans. Image Process. 22(11) (2013) 4422-4435.

15. C. Allain and M. Cloitre, Characterizing the lacunarity of random and deterministic fractal sets, Phys. Rev. A 44(6) (1991) 3552-3558.

16. Q. Chen, Multifractal modeling and lacunarity analysis, Math. Geology 29(7) (1997) 919-932.

17. H. Zhou, E. Perfect, B. G. Li and Y. Z. Lu, Effects of bit depth on the multifractal analysis of grayscale image, Fractals 18 (2010) 127-138.

18. E. Perfect, R. W. Gentry, M. C. Sukop and J. E. Lawson, Multifractal Sierpinski carpets: Theory and application to upscaling effective saturated hydraulic conductivity, Geoderma 134 (2006) 240252.

19. S. R. Koirala, E. Perfect, R. W. Gentryand and J. W. Kim, Effective saturated hydraulic conductivity of two-dimensional random multifractal fields, Water Resour. Res. 48 (2008) 1-9.

20. H. Zhou, E. Perfect, Y. Z. Lu, B. G. Li and X. H. Peng, Multifractal analyses of grayscale and binary soil thin section images, Fractals 19(3) (2011) 1-11.

21. R. E. Plotnick, R. H. Gardner and R. V. O'Neill, Lacunarity indices as measures of landscape texture, Landsc. Ecol. 8(3) (1993) 201-2011.

22. E. Perfect and M. C. Sukop, Models relating solute dispersion to pore space geometry: A review, Soil Sci. Soc. Am. sp. pub 56 (2001) 77-145.

23. J. Grau, V. Méndez, A. M. Tarquis, M. C. Díaz and A. Saa, Comparison of gliding box and boxcounting methods in soil image analysis, Geoderma 134 (2006) 349-359. 